\title{
Tensile properties and drawability of thin bimetallic aluminum- scandium-zirconium / stainless steel foils and monometallic Al- Sc-Zr fabricated by magnetron sputtering
}

\author{
Julien Kovac ${ }^{1, *}$, Lukas Heinrich ${ }^{2}$, Bernd Koehler $^{1}$, Andreas Mehner ${ }^{1}$, Brigitte Clausen ${ }^{1}$ and Hans-Werner Zoch ${ }^{1,3}$ \\ ${ }^{1}$ Leibniz Institute for Materials Engineering IWT, Bremen, Germany \\ ${ }^{2}$ Bremer Institut für angewandte Strahltechnik (BIAS), Bremen, Germany \\ ${ }^{3}$ University of Bremen, Faculty of Production Engineering, Bremen, Germany
}

\begin{abstract}
Al-Sc-Zr alloys are interesting for the production of high strength micro components by micro deep drawing. These alloys show a good hardenability due to the formation of nanometer-scale spheroidal $\mathrm{Al}_{3}(\mathrm{Sc}, \mathrm{Zr})$ precipitates, which are highly coherent with the aluminum matrix. However, the formation of these precipitates in Al-Sc-Zr foils fabricated by conventional metallurgical methods dramatically reduces their ductility and drawability. In this work, magnetron sputtering was used to produce Al-Sc-Zr foils and $\mathrm{Al}-\mathrm{Sc}-\mathrm{Zr} /$ stainless steel bimetallic foils which are nearly free of these precipitates. Tensile tests were carried out to measure and compare the mechanical properties of monometallic Al-Sc-Zr foils and bimetallic Al-Sc-Zr / stainless steel foils deposited with varying plasma target powers and containing different volume fractions (layer thickness) of Al-Sc-Zr. Micro deep drawing was used to determine the drawability of selected monometallic and bimetallic foils. The results show that the density of monometallic Al-Sc-Zr foils can be improved significantly by increasing the DC target power and by using the high power impulse magnetron sputtering (HiPIMS) technology, resulting in foils with higher ductility. Bimetallic foils achieved higher strength and ductility than monometallic Al-Sc-Zr foils. Their mechanical properties vary with the target power and the volume fraction (thickness) of Al-Sc-Zr. The limit drawing ratio of HiPIMS deposited monometallic foil was 1.7 or 1.8 depending on the side of the foil facing the die, whereas a limit drawing ratio of 1.9 was observed for bimetallic foils.
\end{abstract}

Keywords: Micro forming, Sheet metal, PVD-coating

\section{Introduction}

Micro deep drawing is an efficient cold sheet forming process for the mass production of hollow microscopic parts with complex shape, which are widely used in micro system technologies (MST) and micro electromechanical systems (MEMS) such as connector pins, resistor caps, lead chip frames or parts of pressure sensors [1]. The principle of micro deep drawing is essentially similar to the conventional (macroscopic) deep drawing process: a metallic sheet is maintained by a blank holder and put in shape through the action of a punch into a die. However, the microscopic dimensions of the finished parts require the use of thin foils with a thickness generally below $50 \mu \mathrm{m}$ instead of sheets of a few millimeters thickness. This thickness reduction leads to the occurrence of size effects which greatly influence the mechanical and tribological behavior of the drawn foil [2]. Therefore, a considerable research effort is made to improve the efficiency and reliability of micro deep drawing and of other micro forming processes [3]. Foil

\footnotetext{
* Corresponding author: kovac@iwt-bremen.de
}

materials employed in micro deep drawing process are quite restricted to a few types of highly available cold rolled foil strips such as pure copper, pure aluminum or austenitic stainless steel [4] although studies were conducted to investigate the drawability of other special materials $[5,6]$.

Among all the typical alloying elements in aluminum alloys, scandium is one of the most effective elements to improve the mechanical properties. Indeed, the addition of scandium into the aluminum matrix results in the formation of nanometer-scale $\mathrm{Al}_{3} \mathrm{Sc}$ precipitates, which lattice parameters and structure are close to those of the aluminum matrix [7]. This low mismatch ensures a high coherency of the precipitates with the aluminum matrix. Because of their high coherency and dispersivity, the $\mathrm{Al}_{3} \mathrm{Sc}$ precipitates efficiently oppose the mobility of dislocations and grain boundaries, which considerably increases the hardness and the yield strength as well as the recrystallization temperature of the alloy [8]. Beside 
these effects on the mechanical properties, it has also been reported that scandium also improves the corrosion resistance [9] and the weldability [10] of aluminum. Nevertheless, the hardness of Al-Sc alloys also depends on the size of the $\mathrm{Al}_{3} \mathrm{Sc}$ precipitates. During heat treatment, the precipitates may grow up to a critical radius at which, the interface between the precipitates and the matrix becomes incoherent [11]. Consequently, dislocations and grain boundaries are less efficiently pinned, and the hardness of the alloy starts to decrease.

This issue can be addressed by the addition of zirconium to the Al-Sc alloys. As a result, small amounts of zirconium are incorporated into $\mathrm{Al}_{3} \mathrm{Sc}$ precipitates. $\mathrm{Al}_{3}\left(\mathrm{Sc}_{1-\mathrm{x}} \mathrm{Zr}_{\mathrm{x}}\right)$ precipitates are more stable and coarsen slower due to the lesser diffusivity of $\mathrm{Zr}$ in aluminum [12]. Moreover, substantial augmentations of strength have been reported in $\mathrm{Al}-\mathrm{Sc}-\mathrm{Zr}$ alloys compared to zirconium free Al-Sc alloys [13].

Due to their excellent mechanical properties, Al-Sc$\mathrm{Zr}$ alloys can be employed for deep drawing high strength micro components for mechanically demanding applications. For instance, body parts of micro valves may be submitted to large fluid or contact pressures from other parts of the valve and therefore, the body has to sustain relatively large elastic deformations and avoid permanent plastic deformation. Due to the combination of high yield strength and low Young's modulus, Al-Sc$\mathrm{Zr}$, can achieve larger elastic deformations than austenitic stainless steel. Moreover Al-Sc-Zr alloys show a higher resistance to fatigue then other conventional aluminum alloys [14] and may thus be used to improve the reliability of micro parts, since a considerable demand exists in the industry [15].

Drawing high strength Al-Sc-Zr alloys is however, not an easy task since precipitates can dramatically reduce the ductility and thus, the formability of these alloys. Furthermore, the formation of precipitates is difficult to avoid in cast alloys, due to the low maximum solubility of $\mathrm{Sc}$ and $\mathrm{Zr}$ in aluminum. Therefore, alternative methods were developed to prepare thin precipitates free $\mathrm{Al}-\mathrm{Sc}-\mathrm{Zr}$ foils for micro deep drawing. In previous investigations magnetron sputtering was successfully used to prepare freestanding $\mathrm{Al}-\mathrm{Zr}$ foils [16]. Their microstructure consisted of a nearly precipitate free oversaturated aluminum solid solution which could be hardened by heat treatment after deep drawing [17]. The limit drawing ratio of these foils was 1.7 , which is comparable to conventional pure aluminum foils [18]. This was less than other materials like copper or stainless steel, which drawability is higher.

One possible way to increase the formability of aluminum or aluminum alloy foils would be to bond these foils to materials with better formability like stainless steel and thus making bimetallic foils instead of monometallic foils. Bimetallic sheets are composed of a stacking of layers of two different materials. Usually one of the materials is hard and brittle, as the other is soft and ductile and the mechanical properties of bimetallic sheets are a compromise of those of the components. More precisely, it was shown that some properties of a bimetallic sheet can be deduced from the rule of mixtures, which means that the property of the bimetallic sheet is an average value of the properties of the respective components weighted by their volume fractions. Hence, in aluminum / stainless steel bimetallic sheets, the apparent elastic modulus, the tensile strength and the plastic strength coefficient (according to Hollomon's law) follow the rule of mixtures, whereas the uniform elongation and the strain hardening exponents could be deduced by a force weighted law of mixtures [19]. In every case, a significant improvement of strength and ductility compared to pure aluminum was observed. The deep drawing behavior of aluminum / stainless steel bimetallic sheets was investigated by various authors. Parsa et al. investigated the evolution of the limit drawing ratio in single drawing and redrawing for bi-layered sheets with various proportions of aluminum and stainless steel through simulation and experiments [20]. They found that with increasing steel layer thickness (i. e. decreasing aluminum layer thickness) the limit drawing ratio increased. However, the limit drawing ratio depended on the experimental setup and was lower when the aluminum layer faced toward the punch. A similar behavior was obtained by simulation by Takuda et al. [21] and Afshin et al. in warm deep drawing [22].

To the best of our knowledge, up to now only thick (around $1 \mathrm{~mm}$ ) bimetallic sheets were obtained by the conventional methods like roll bonding [23]. In this study, direct current magnetron sputtering (DCMS) and high-power impulse magnetron sputtering (HiPIMS) are used to produce $30 \mu \mathrm{m}$ thick monometallic $\mathrm{Al}-\mathrm{Sc}-\mathrm{Zr}$ foils and two layer bimetallic Al-Sc-Zr / stainless steel foils under various conditions. Tensile testing was carried out to compare their mechanical properties and micro deep drawing experiments were made to compare their drawability.

\section{Experimental methods}

\subsection{Preparation of $\mathrm{Al}-\mathrm{Sc}-\mathrm{Zr}$ monometallic foils and Al-Sc-Zr / stainless steel bimetallic foils by magnetron sputtering}

Different monometallic Al-Sc-Zr foils and bilayer Al-Sc$\mathrm{Zr} /$ steel foils were produced by DCMS and HiPIMS. For the production of thin $(\approx 30 \mu \mathrm{m})$ monometallic Al$\mathrm{Sc}-\mathrm{Zr}$ foils, different $\mathrm{Al}-\mathrm{Sc}-\mathrm{Zr}$ alloys with $\approx 1.8$ mass- $\%$ Sc and $\approx 0.25$ or 0.6 mass- $\% \mathrm{Zr}$ were deposited onto $100 \mu \mathrm{m}$ polished sheets $\left(1300 \times 150 \mathrm{~mm}^{2}\right)$ of low carbon steel. The steel substrates were removed by etching in concentrated nitric acid after the deposition process. The nitric acid did not attack the remaining freestanding Al$\mathrm{Sc}-\mathrm{Zr}$ foils. Nevertheless hydrogen absorption and diffusion might affect the mechanical properties of the foils. For the production of bimetallic foils, Al-Sc-Zr coatings with thicknesses between 5 and $15 \mu \mathrm{m}$ were 
deposited onto commercial austenitic stainless steel foils (X5CrNi18-10: 8 mass-\% Cr, 10 mass- $\% ~ N i$ and 0.05 mass- $\%$ C). The length of the stainless steel strips was $1.3 \mathrm{~m}$ and the width was $50 \mathrm{~mm}$. The thickness of the substrate strips was chosen between $15 \mu \mathrm{m}, 10 \mu \mathrm{m}$ and $25 \mu \mathrm{m}$ according to the Al-Sc-Zr coating thickness, so that the thickness of the resulting bimetallic foils was always close to $30 \mu \mathrm{m}$.

The deposition process was carried out in a commercial CemeCon CC800/9 magnetron sputtering unit fitted with four DC cathodes and two HIPIMS cathodes. For direct current magnetron sputtering (DCMS) processes, three of the DC cathodes were fitted with aluminum targets alloyed with 2 mass-\% Sc and the remaining DC cathode was fitted with an aluminum target alloyed with 2 mass- $\%$ zirconium as depicted in Figure 1a. For HiPIMS processes the two HiPIMS cathodes were fitted with two copper-bonded aluminum targets alloyed with 1.5 mass- $\%$ Sc 0.5 mass- $\% \mathrm{Zr}$. (Figure 1b). The dimensions of the targets were $488 \times 88$ $\times 9 \mathrm{~mm}^{3}$. A homogeneous alloying element distribution is ensured by setting the different substrate foils around a rotating substrate table (Figure 2), which rotation speed was set to 1 rotation per minute. The distance between the different targets and the substrate was in every case about $50 \mathrm{~mm}$.

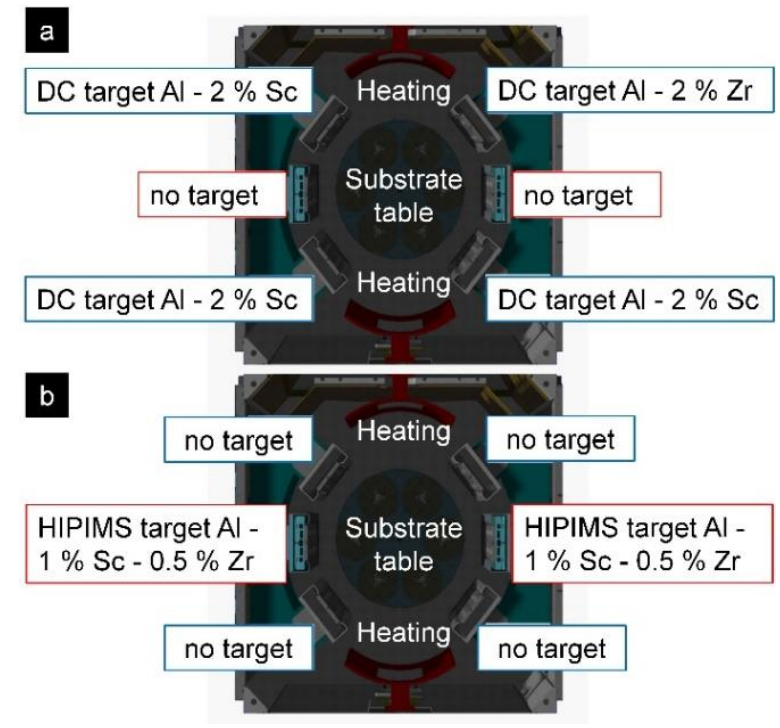

Fig. 1. Target setup in the magnetron sputtering unit for the production of $\mathrm{Al}-\mathrm{Sc}-\mathrm{Zr}$ monometallic foils and $\mathrm{Al}-\mathrm{Sc}-\mathrm{Zr}$ / stainless steel bimetallic foils: a) setup for DCMS, b) setup for HIPIMS.

All deposition processes were carried out as follows: First, the different substrates were cleaned with ethanol and fixed to the substrate table. Then, the deposition chamber was evacuated up to a residual air pressure of about $510^{-7}$ mbar. Then, the substrate was heated for 2 hours to evaporate traces of humidity. Next, a flow of $250 \mathrm{sccm}$ of argon and $25 \mathrm{sccm}$ of krypton has been let into the chamber and the substrate table was biased with a middle frequency (MF) voltage of $160 \mathrm{~V}$ at a frequency of $650 \mathrm{kHz}$. Consequently, plasma is created, and the substrate is bombarded with accelerating argon and krypton ions, which remove impurities from the substrate surface. This ion etching phase was carried out for 1 hour. Afterwards, the substrate was cooled down for 3 hours with the same constant flow of argon. Finally, for the deposition process, the argon pressure was set to $310^{-4}$ mbar. The different cathodes were biased with negative voltage, whereas the substrate and the rest of the unit parts were earthed. Plasma of positive argon ions and electrons was created, and argon ions were accelerated to the target which results in the sputtering (vaporization) of the target atoms which diffuse through the chamber and condense on the substrate. The coating thickness was varied by adapting the deposition time.

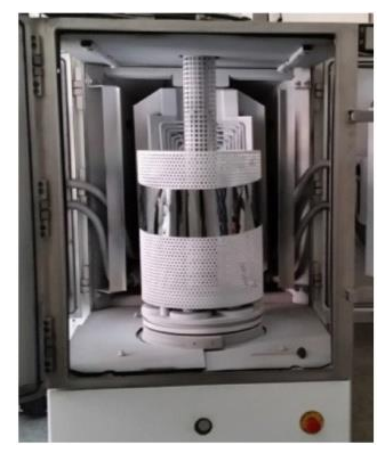

Fig. 2. Picture of the rotating substrate table in the magnetron sputtering unit fitted with a polished steel sheet substrate.

With this setup three different monometallic Al-Sc$\mathrm{Zr}$ foils and 5 different Al-Sc-Zr / stainless steel bimetallic foils were produced. Among the three monometallic foils, two foils were deposited with DC power with target powers at respectively $2 \mathrm{~kW}$ for 18000 seconds and $4 \mathrm{~kW}$ for 9000 seconds. In this case a constant voltage is continuously applied. The third variant was deposited with HiPIMS. The average target power was set up to $2 \mathrm{~kW}$ and the deposition time was 50000 seconds. In this case the voltage varies periodically. During off-phase the target is not biased, and the current is loaded in a capacitor and released during the duty phase. The time of the duty cycle was $200 \mu$ s (20\% of the period) and the frequency was 1000 $\mathrm{Hz}$. Three of the bimetallic foils consist of $5 \mu \mathrm{m}$ of Al$\mathrm{Sc}-\mathrm{Zr}$ and $25 \mu \mathrm{m}$ of stainless steel. The Al-Sc-Zr layer deposition was done with similar target powers (respectively $2 \mathrm{~kW}$ DC, $4 \mathrm{~kW} \mathrm{DC}$ and $2 \mathrm{~kW}$ HiPIMS) as for the monometallic foils. Two supplemental bimetallic foils were deposited at $4 \mathrm{~kW}$ DC with different layer thicknesses, respectively $10 \mu \mathrm{m} \mathrm{Al-Sc-Zr} \mathrm{/} 20 \mu \mathrm{m}$ stainless steel and $15 \mu \mathrm{m}$ Al-Sc-Zr / $15 \mu \mathrm{m}$ stainless steel. The characteristics of the different monometallic and bimetallic foils are summarized in Table 1.

The chemical composition of the different Al-Sc-Zr foils was measured by glow discharge optical emission spectroscopy GDOES (LECO GDS-750A). The results are summarized in table 2 . 
Table 1. Data of the different monometallic Al-Sc-Zr foils and of the different Al-Sc-Zr / stainless steel bimetallic foils.

\begin{tabular}{|c|c|c|c|}
\hline $\begin{array}{c}\text { Specim } \\
\text { en }\end{array}$ & $\begin{array}{c}\text { Al-Sc-Zr } \\
\text { layer } \\
\text { thickness } \\
{[\mu \mathrm{m}]}\end{array}$ & $\begin{array}{c}\text { Stainless } \\
\text { steel layer } \\
\text { thickness } \\
{[\mu \mathrm{m}]} \\
\end{array}$ & $\begin{array}{c}\text { Target power and } \\
\text { deposition time }\end{array}$ \\
\hline MDC2 & 28.8 & 0 & DC $2 \mathrm{~kW} 18000 \mathrm{~s}$ \\
\hline MDC4 & 26.8 & 0 & DC $4 \mathrm{~kW} 9000 \mathrm{~s}$ \\
\hline MHP & 28.7 & 0 & HIPIMS $2 \mathrm{~kW} 50000 \mathrm{~s}$ \\
\hline BDC2 & 5 & 25 & DC 2 kW $3168 \mathrm{~s}$ \\
\hline $\mathrm{BDC} 4 \mathrm{a}$ & 5 & 25 & DC $4 \mathrm{~kW} 1677 \mathrm{~s}$ \\
\hline $\mathrm{BDC} 4 \mathrm{~b}$ & 10 & 20 & DC $4 \mathrm{~kW} 3378 \mathrm{~s}$ \\
\hline $\mathrm{BDC} 4 \mathrm{c}$ & 15 & 15 & DC $4 \mathrm{~kW} 5000 \mathrm{~s}$ \\
\hline BHP & 5 & 25 & HIPIMS $2 \mathrm{~kW} 8881 \mathrm{~s}$ \\
\hline
\end{tabular}

Table 2. Chemical compositions of the different monometallic Al-Sc-Zr foils.

\begin{tabular}{|c|c|c|c|}
\hline Foil & mass-\% Al & mass-\% Sc & mass-\% Zr \\
\hline MDC2 & Balance & 1.82 & 0.26 \\
\hline MDC4 & Balance & 1.87 & 0.25 \\
\hline MHP & Balance & 1.78 & 0.57 \\
\hline
\end{tabular}

\subsection{Determination of the tensile properties and other characteristics}

The tensile properties of the different monometallic and bimetallic foils and of a $30 \mu \mathrm{m}$ thick stainless steel foil were determined using special tensile test specimens which were produced by cutting each foil into 10 rectangular pieces $\left(50 \times 10 \mathrm{~mm}^{2}\right)$. The ends of the foil pieces were reinforced by sticking four pieces of $200 \mu \mathrm{m}$ of steel as illustrated in figure 3 . The testing unit was an INSTRON E1000 tensile tester which is adapted for the measurement of low thickness specimens. During testing, load and strain were measured by a calibrated $1000 \mathrm{~N}$ load cell meeting the requirements of ISO 75001 and ASTM E4 and a video extensometer, respectively. Additional details about the unit can be found in [24]. The average values of elastic modulus, yield strength, tensile strength and fracture strain of each foil variant were determined from 10 specimens.

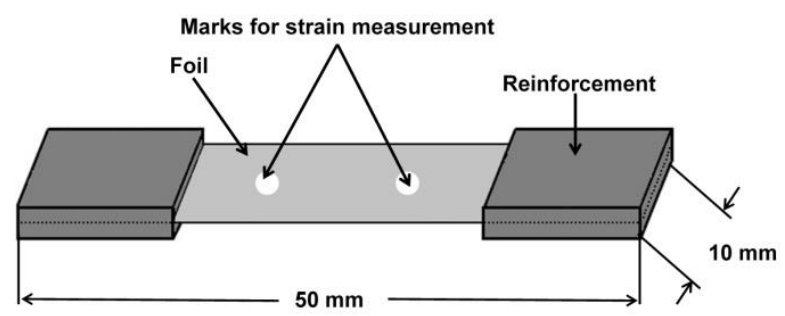

Fig. 3. Sketch of a tensile specimen.

The morphology of the foils was investigated by scanning electron microscopy (SEM) (Camscan
MV2300, EO Scan). Transmission electron microscopy was used to determine the presence of precipitates (Phillips CM12).

\subsection{Determination of the formability in micro deep drawing}

The geometric data of the tools are summarized in table 3. A sketch of the used setup can be seen in Figure 4 . The deep drawing process was carried out on a single axis micro forming press with a maximum punch force of $500 \mathrm{~N}$ and a constant punch velocity of $10 \mathrm{~mm} / \mathrm{s}$ using HBO $947 / 11$ as lubricant. The punch force was measured with a Kistler 9217A piezo load cell with an accuracy of $0.01 \mathrm{~N}$. Punch displacement was measured with a Heidenhain LS477 linear scale with an accuracy of $1 \mu \mathrm{m}$. The press is driven by a servo motor controlled by a NI 9514 servo drive interface. The blank holder acts passively. It uses its own weight and is supported by two springs. Blank holder pressure can be adjusted by changing the spring tension and was set to $\mathrm{P}_{\mathrm{NHD}}=5 \mathrm{MPa}$. The blank positioning was realized by an automated positioning system. The blank was positioned with a pneumatic gripper that is driven by a linear direct drive cross table. The position of the blank was then measured using an Allied Vision G 917 B monochrome CCD camera with a resolution of $9 \mathrm{Mpx}$ equipped with a telecentric lens with built-in coaxial illumination and a magnification of 0.75 . With this setup the blanks can be positioned within a radius of $10 \mu \mathrm{m}$ from the center of the drawing die.

Table 3. Specifications of tool geometry used in experiment.

\begin{tabular}{|l|r|}
\hline Punch diameter (mm) & 1.005 \\
\hline Punch radius (mm) & 0.110 \\
\hline Drawing die diameter (mm) & 1.061 \\
\hline Average drawing die radius (mm) & 0.077 \\
\hline Drawing gap (mm) & 0.028 \\
\hline
\end{tabular}

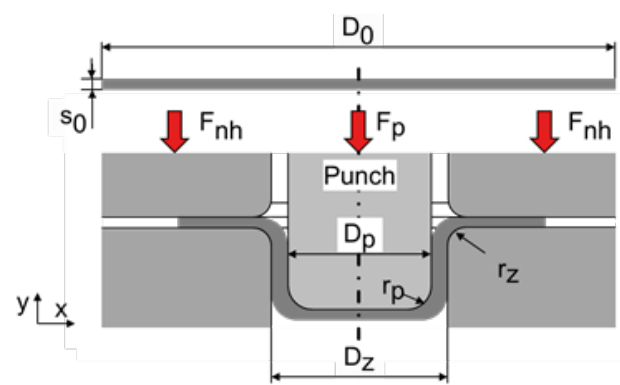

Blank

Blank holder

Heinrich 2016

Fig. 4. Sketch of the deep drawing setup.

\section{Results and discussion}

\subsection{Properties of monometallic Al-Sc-Zr foils and $\mathrm{Al}-\mathrm{Sc}-\mathrm{Zr} / \mathrm{stainless}$ steel bimetallic foils}

3.1.1 Morphological and microstructural characteristics of monometallic and bimetallic foils. 
The morphology of the different monometallic foils after deposition and separation from substrate has been investigated by scanning electron microscopy. Pictures of both the cross section and free surface of the different monometallic foils are shown in Figure 5.

The foils show the classical columnar morphology of sputter deposited coatings [25]. This implicates that monometallic sputtered Al-Sc-Zr foils present two different surfaces. The bottom surface, which was in contact with the substrate, usually consists of tiny close packed amorphous grains, and this surface reflects the topology of the substrate. At the top surface (in Figure 5) or free surface, the columnar crystallites grow freely and the tapered shape of these crystallites results in a rough surface. The foil sputtered with $2 \mathrm{~kW}$ DC power (MDC2) shows a relatively dense morphology in the bottom region near the substrate contact surface. About $10 \mu \mathrm{m}$ beneath the free surface however, the boundaries between the columnar crystallites are more open voided and the foil is more porous. In comparison, the foil produced at $4 \mathrm{~kW}$ (MDC4) and the HiPIMS deposited foil (MHP) are overall denser since porosities are less frequent and confined to the near free surface region. This observation was confirmed by optical microscopy observations of the cross sections of the foils which are featured on Figure 6.
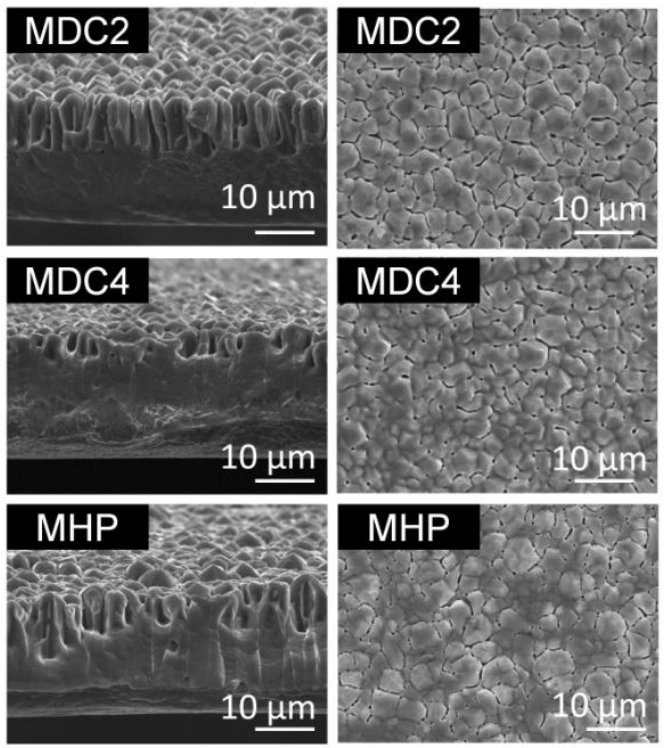

Fig. 5. Scanning electron microscopy pictures of the cross section (left) and free surface (right) of the different monometallic Al-Sc-Zr foils.

This increase of density is to be related to the higher kinetic energy of the sputtered atoms when the target power is at $4 \mathrm{~kW}$. Indeed, when the target power increases, the voltage at the target increases as well and the argon ions striking the targets have a higher kinetic energy. This higher kinetic energy is also transmitted to the sputtered atoms. The mobility of the atoms impinging on the coating surface, is higher and the voids in the coating are more likely to be filled [26]. The same mechanism can be applied to the HiPIMS deposited foil
(MHP). Indeed, in high power impulse magnetron sputtering, the average value of the target power is only $2 \mathrm{~kW}$, while the energy of the pulse may reach more than $10 \mathrm{~kW}[26]$.

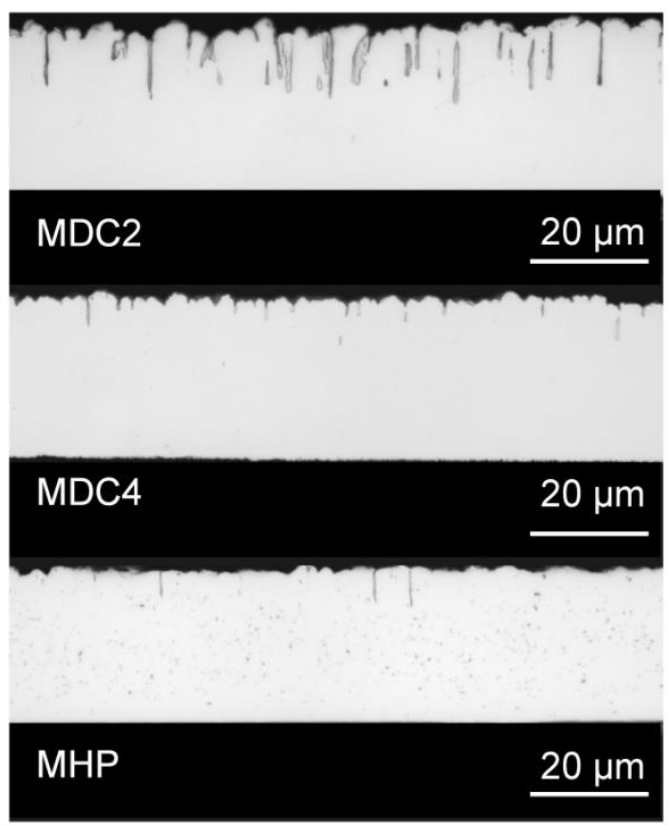

Fig. 6. Optical microscopy pictures of the cross sections of the different $\mathrm{Al}-\mathrm{Sc}-\mathrm{Zr}$ monometallic foils after embedding and polishing.

Compared to monometallic foils, scanning electron microscopy observations carried out on bimetallic foils (Figure 7) show a finer and denser columnar morphology. This morphology can be explained by the thinner Al-Sc-Zr coating thickness in the bimetallic foils. The diameter of the columnar crystallites increases when the coating grows. Porosities are the result of shadowing effect from already formed crystallites, which prevent impinging atoms to cover homogeneously the whole surface. Since the top diameter of crystallites is smaller in thin coatings, it can be assumed that shadowing effects are less severe for thin coatings, which results in les surface porosities than in thick coatings.

Transmission electron microscopy was used to determine the phase content of the Al-Sc-Zr foils after deposition and after heat treatment at $500{ }^{\circ} \mathrm{C}$ for 1 hour for comparison purpose. Pictures and the corresponding electron diffraction patterns taken according to $\left\{\begin{array}{lll}1 & 0 & 0\end{array}\right\}$ zone axis are featured in Figure 8. After deposition, no $\mathrm{Al}_{3}(\mathrm{Sc}, \mathrm{Zr})$ precipitates are visible on the picture and only reflections corresponding to the face centered cubic lattice of the $\alpha$-aluminum phase are to be found on the diffraction pattern. However, after heat treatment, precipitates with diameters between 30 and $40 \mathrm{~nm}$ are clearly visible on the picture and besides the $\alpha$ aluminum; further reflections can be identified on the pattern. These have a structure in agreement with the simple cubic $\mathrm{L}_{2}$ structure of $\mathrm{Al}_{3}(\mathrm{Sc}, \mathrm{Zr})$ precipitates. These results confirm that Al-Sc-Zr foils elaborated by magnetron sputtering process consist of an oversaturated 
a-aluminum solution as for $\mathrm{Al}-\mathrm{Sc}$ and $\mathrm{Al}-\mathrm{Zr}$ alloys in a previous work [16].

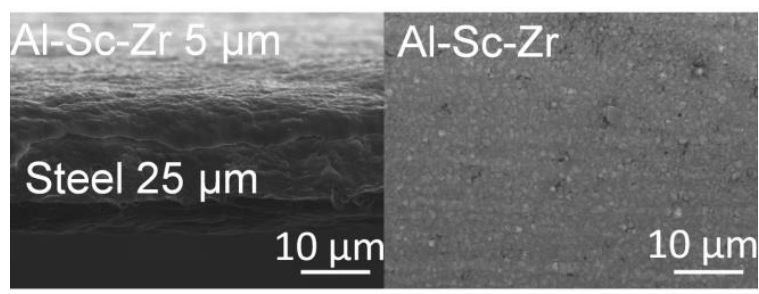

Fig. 7. Scanning electron microscopy pictures of the cross section (left) and free surface (right) of a bimetallic Al-Sc-Zr (5 $\mu \mathrm{m}) /$ Steel $(25 \mu \mathrm{m})$ foil.

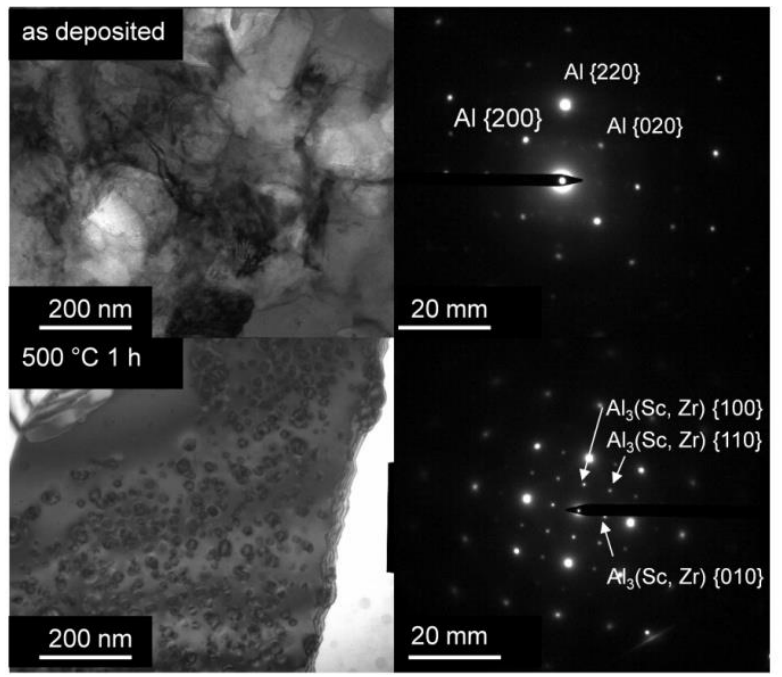

Fig. 8. Transmission electron microscopy pictures (left) and electron diffraction patterns (right) of a sputtered Al-Sc-Zr foil after deposition and after heat treatment for $1 \mathrm{~h}$ at $500{ }^{\circ} \mathrm{C}$. Camera constant: $4,233 \mathrm{~nm} \times \mathrm{mm}$, zone axis $\left\{\begin{array}{lll}1 & 0 & 0\end{array}\right\}$.

\subsubsection{Tensile properties of monometallic Al-Sc-Zr} foils.

The mechanical properties of the monometallic Al$\mathrm{Sc}-\mathrm{Zr}$ foils produced with different DC and HIPIMS powers were determined by tensile tests. Various mechanical properties, namely the Young's modulus (E), the $0.01 \%$ offset yield strength $\left(\mathrm{R}_{\mathrm{p} 0.01}\right)$, the ultimate tensile strength $\left(\mathrm{R}_{\mathrm{m}}\right)$ as well as the deformation at fracture (A) were extracted from the data. The average values for 10 specimens for each foil are summarized in Table 4. Selected tensile tests curves of each foil are featured in Figure 9.

A foil sputtered at $2 \mathrm{~kW}$ DC power (MDC2) shows a relatively moderate tensile strength of $292 \mathrm{MPa}$ and fails at only $0.58 \%$ strain. Furthermore, its elastic modulus is lower than the expected value of $70 \mathrm{GPa}$ for aluminum. These poor mechanical properties are explained by the high porosity of the foil as shown in Figures 4 and 5. When the DC power is increased up to $4 \mathrm{~kW}$ (MDC4), the kinetic energy of the impinging species increases as well as the mobility of the atoms on the coating surface and the porosities are more efficiently filled [26]. The denser structure of this foil improves its ductility, tensile strength and Young's modulus, which values reach respectively $1.45 \%, 314 \mathrm{MPa}, 400 \mathrm{MPa}$ and $69 \mathrm{GPa}$. The strain at fracture remains however under $2 \%$, which can be related to the high yield and tensile strength of the foil. This higher strength is also the consequence of the higher energy of the vapor atoms. Indeed, the higher energy of the vapor atoms may also results in impulse driven displacements in the coating and therefore increases the density of dislocations and points defects of the coating [28]. The foil prepared with HiPIMS process shows by contrast much more interesting characteristics. Its average ductility is much higher than in the DC foils. Young's modulus is higher than in the foil MDC2. The yield and tensile strengths are nevertheless weaker than in the other foils. In the used HiPIMS process the peak power may reach $10 \mathrm{~kW}$ which is far more than in the DCMS process. According to the above mentioned mechanism a higher density is expected too.

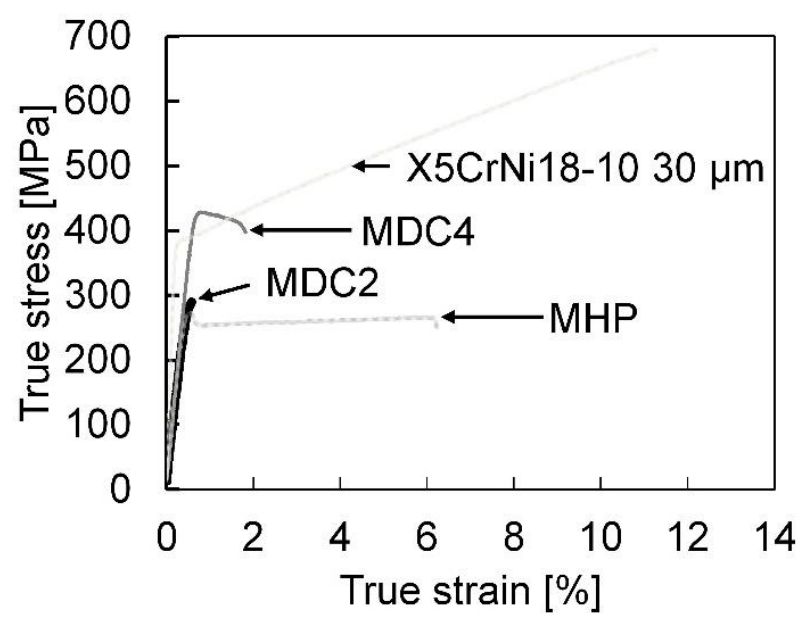

Fig. 9. Selected tensile test curves of each of the produced Al$\mathrm{Sc}-\mathrm{Zr}$ foil and of a commercial $30 \mu \mathrm{m}$ stainless steel foil.

Table 4. Average values and standard deviations of the mechanical properties of Al-Sc-Zr foils and of a $30 \mu \mathrm{m}$ stainless steel foil.

\begin{tabular}{|c|c|c|c|c|}
\hline Foil & E [GPa] & $\begin{array}{c}\text { Rpo.01 } \\
{[\mathbf{M P a}]}\end{array}$ & $\begin{array}{c}\text { Rm } \\
{[\mathbf{M P a}]}\end{array}$ & A [\%] \\
\hline MDC2 & $58 \pm 4$ & $269 \pm 42$ & $292 \pm 63$ & $0.6 \pm 0.2$ \\
\hline MDC4 & $69 \pm 2$ & $314 \pm 29$ & $400 \pm 38$ & $1.5 \pm 1.3$ \\
\hline MHP & $65 \pm 1$ & $230 \pm 9$ & $260 \pm 12$ & $5.7 \pm 3.9$ \\
\hline Steel & $197 \pm 25$ & $355 \pm 13$ & $684 \pm 41$ & $11.5 \pm 1.8$ \\
\hline
\end{tabular}

\subsubsection{Tensile properties of bimetallic Al-Sc-Zr / stainless steel foils.}

In total, five different bimetallic foils were produced. Three of them (BDC2, BDC4a and BHP) consist of a 25 $\mu \mathrm{m}$ thick stainless steel substrate foil coated with $5 \mu \mathrm{m}$ of sputtered $\mathrm{Al}-\mathrm{Sc}-\mathrm{Zr}$ and were produced in the same conditions as the three monometallic Al-Sc-Zr foils in 
order to properly compare the mechanical properties of bimetallic foils to those of monometallic foils and to check if the changing mechanical properties of the differently deposited Al-Sc-Zr layers also affect the properties of the Al-Sc-Zr / stainless steel bimetallic foils. In two other bimetallic foils (BDC4b and BDC4c) the stainless steel and Al-Sc-Zr layer thicknesses were varied at respectively $10 \mu \mathrm{m}$ of Al-Sc-Zr for $20 \mu \mathrm{m}$ stainless steel and $15 \mu \mathrm{m}$ of Al-Sc-Zr for $15 \mu \mathrm{m}$ of stainless steel. These bimetallic foils were produced in the same conditions as the foil BDC4a so that the validity of the law of mixture can be tested.

The mechanical properties of the different bimetallic foils were evaluated by tensile testing in the same way as for monometallic Al-Sc-Zr foils. Exemplary tensile test curves of each of the considered bimetallic foils are featured on Figure 10. For comparison purpose, exemplary tensile test curves of the monometallic Al-Sc$\mathrm{Zr}$ sputtered at $4 \mathrm{~kW}$ DC and of a $30 \mu \mathrm{m}$ thick stainless steel foil were also featured on the same figure. The average values and standard deviations of the mechanical properties of the bimetallic foils over 10 tensile specimens are summarized in Table 5.

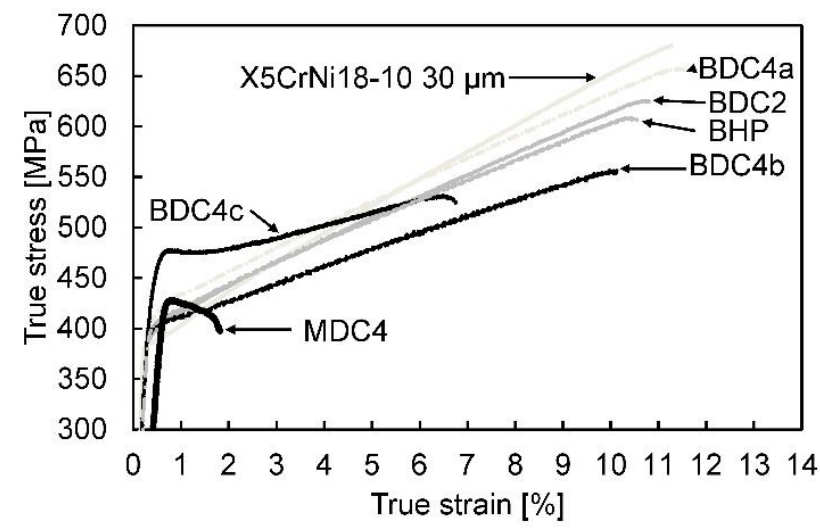

Fig. 10. Selected tensile test curves of each of the produced Al$\mathrm{Sc}-\mathrm{Zr}$ / stainless steel bimetallic foils, of a commercial $30 \mu \mathrm{m}$ stainless steel foil and of a monometallic Al-Sc-Zr foil sputtered at $4 \mathrm{~kW}$ DC (MDC4)

The bimetallic foils BDC2, BDC4a and BHP sputtered at respectively $2 \mathrm{~kW} \mathrm{DC,} 4 \mathrm{~kW}$ DC and $2 \mathrm{~kW}$ HiPIMS show much higher apparentYoung's modulus, deformations at fracture, yield and tensile strengths than the three monometallic Al-Sc-Zr foils produced with similar parameters (MDC2, MDC4, MHP). Furthermore, by comparing the properties of these three bimetallic foils, one can notice that apparent Young's modulus and the tensile strength of these bimetallic foils vary similarly as Young's modulus and tensile strengths of their corresponding monometallic foils. Hence. by increasing the target power from 2 to $4 \mathrm{~kW} \mathrm{DC}$, both the apparent elastic modulus and tensile strength increase. The bimetallic foil BHP shows an intermediary apparent Young's modulus of $180 \mathrm{GPa}$ and a lower tensile strength than the bimetallic foils BDC2 and BDC4a, which is also the case for the monometallic foil MHP compared to MDC2 and MDC4. This similar trend between monometallic and bimetallic foils seems less evident for the yield strength, whereas for the deformation at fracture, the trend observed for monometallic foils is not reproduced in bimetallic foils. In each bimetallic foil, the deformation at fracture approximates the deformation at fracture of the monometallic stainless steel foil (Table 4).

The comparison of the mechanical properties of the bimetallic foils BDC4a, BDC4b and BDC4c sputtered at $4 \mathrm{~kW}$ DC and containing Al-Sc-Zr layers of respectively $5 \mu \mathrm{m}, 10 \mu \mathrm{m}$ and $15 \mu \mathrm{m}$, shows as expected, that the apparent Young's modulus, the yield and tensile strength of the bimetallic foils increase when the volume fraction (ratio of the Al-Sc-Zr layer thickness to the total thickness of the bimetallic foil) of Al-Sc-Zr in the bimetallic foil decrease or reciprocally when the volume fraction of stainless steel increases. The evolution of the mechanical properties in bimetallic foils with the volume fraction of components can be predicted by the rule of mixtures [19]:

$$
P_{\mathrm{AB}}=V_{\mathrm{A}} P_{\mathrm{A}}+V_{\mathrm{B}} P_{\mathrm{B}}(1)
$$

With $V_{A}$ and $V_{B}$ designating the volume fractions of component $\mathrm{A}$ and $\mathrm{B}$ in the bimetallic foil and $\mathrm{P}_{\mathrm{A}}$ and $\mathrm{P}_{\mathrm{B}}$ representing a particular mechanical characteristic of components A and B like Young's modulus, yield strength, strain at fracture or the ultimate tensile strength. The validity of the law of mixture for the prediction of respectively apparent Young's modulus, the deformation at fracture, the yield and the ultimate tensile strength of the produced Al-Sc-Zr / stainless steel bimetallic foils has been tested by using the data of Tables 3 and 4 . Therefore, the theoretical evolution of the different characteristics of the bimetallic foils were predicted from the measured characteristics of monometallic Al-Sc-Zr and stainless steel foils (Table 3) by taking their standard deviations in account, which results in the different plots featured on Figure 10. The experimental characteristics measured in bimetallic foils $\mathrm{BDC} 4 \mathrm{a}, \mathrm{BDC} 4 \mathrm{~b}$ and $\mathrm{BDC} 4 \mathrm{c}$ containing respectively $16.7,33.3$ and $50 \%$ of Al-Sc-Zr were also featured on the different diagrams on Figure 11.

As shown, the rule of mixtures is statistically in quite good agreement with the experimentally extracted yield and tensile strengths of the produced bimetallic foils. Apparent Young's modulus shows a slightly positive deviation from the rule of mixtures, which can be attributed to the higher density of the Al-Sc-Zr layer (Fig. 7) in bimetallic sheet due to the lower thickness of the Al-Sc-Zr coating in bimetallic foils, as previously explained. Finally, the experimental values of the deformation at fracture are not in good agreement with the rule of mixtures. 
Table 5. Average values and standard deviations of the mechanical properties of Al-Sc-Zr / stainless steel bimetallic foils.

\begin{tabular}{|c|c|c|c|c|}
\hline Foil & E [GPa] & $\begin{array}{c}\mathbf{R}_{\mathbf{p 0 . 0 1}} \\
{[\mathbf{M P A}]}\end{array}$ & $\begin{array}{c}\mathbf{R}_{\mathbf{m}} \\
{[\mathbf{M P a}]}\end{array}$ & $\mathbf{A}[\mathbf{\%}]$ \\
\hline BDC2 & $175 \pm 17$ & $323 \pm 17$ & $626 \pm 35$ & $12 \pm 1.3$ \\
\hline BDC4a & $192 \pm 8$ & $339 \pm 12$ & $662 \pm 22$ & $12.6 \pm 1.1$ \\
\hline BDC4b & $177 \pm 14$ & $327 \pm 9$ & $548 \pm 10$ & $12.1 \pm 3$ \\
\hline BDC4c & $148 \pm 25$ & $329 \pm 43$ & $526 \pm 21$ & $11.1 \pm 8$ \\
\hline BHP & $180 \pm 19$ & $347 \pm 26$ & $572 \pm 21$ & $10.8 \pm 1.2$ \\
\hline
\end{tabular}
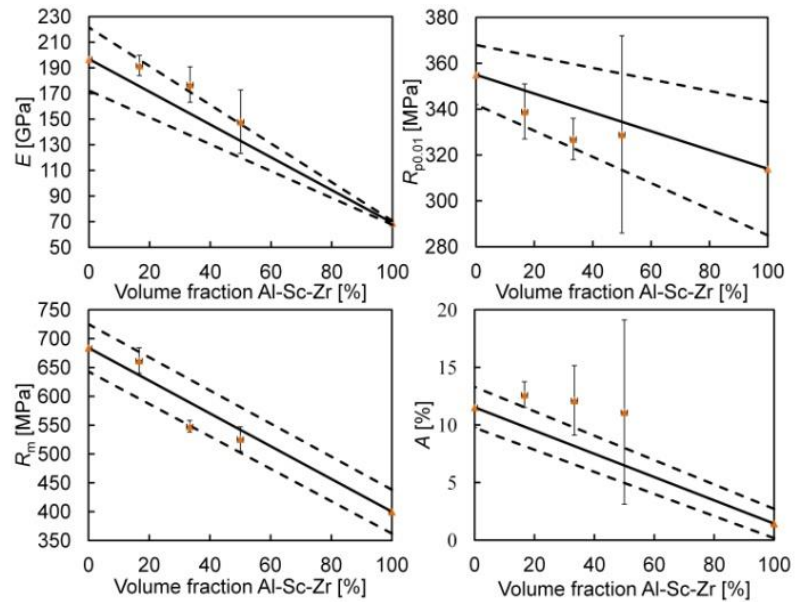

Fig. 11. Comparison of the experimental values of apparent Young's modulus, the yield and tensile strengths and deformation at fractures measured in bimetallic foils BDC4a, $\mathrm{BDC} 4 \mathrm{~b}$ and $\mathrm{BDC} 4 \mathrm{c}$ with those predicted by the law of mixtures. Full line: law of mixture calculated from the average values for pure Al-Sc-Zr and stainless steel in Table 3. Dotted lines: standard deviations of the law of mixtures. Square marks: experimental points.

Broken tensile test specimens of the different bimetallic foils were examined with scanning electron microscopy after tensile testing. As depicted in Figure 12 , the Al-Sc-Zr coating presents no crack indicating a premature coating failure during the tensile testing of the bimetallic foils, despite being submitted to deformations well above the deformation at fracture of monometallic $\mathrm{Al}-\mathrm{Sc}-\mathrm{Zr}$ foils. In other terms, this proves that lamination dramatically improve the ductility of Al-Sc-Zr foils. According to Choi et al., this improvement of mechanical properties is due to warping of the tensile specimen during tensile testing as a consequence of lateral stress resulting from the different contraction behaviors of aluminum and steel [29]. By making the assumption that the aluminum and steel layers are strongly bonded together and that consequently, the axial strain in both layers is equal, these authors could show the validity of the rule of mixture for the prediction of the mechanical properties of bimetallic sheets.

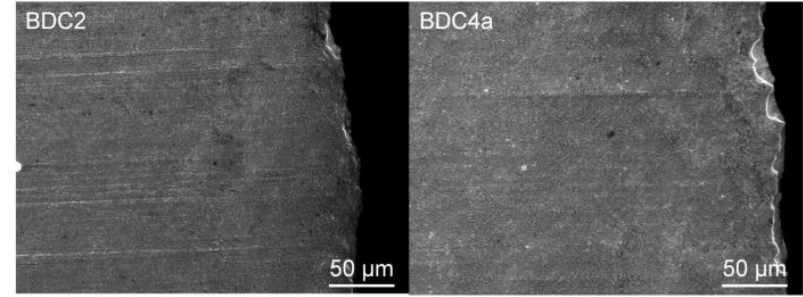

Fig. 12. Scanning electron microscopy pictures of the Al-Sc-Zr free surface of the bimetallic foils $\mathrm{BDC} 2$ and $\mathrm{BDC} 4 \mathrm{a}$ after tensile testing.

\subsection{Micro deep drawing}

Deep drawing experiments were carried out on monometallic Al-Sc-Zr foils and a bimetallic Al-Sc-Zr / stainless steel foil produced by HiPIMS in order to determine the drawability of the foils. As shown in the exemplary process window in Figure 13 a limiting drawing ratio of 1.9 was reached by the bimetallic Al$\mathrm{Sc}-\mathrm{Zr} /$ stainless steel foil (BHP). In this case the stainless steel side was facing the punch.

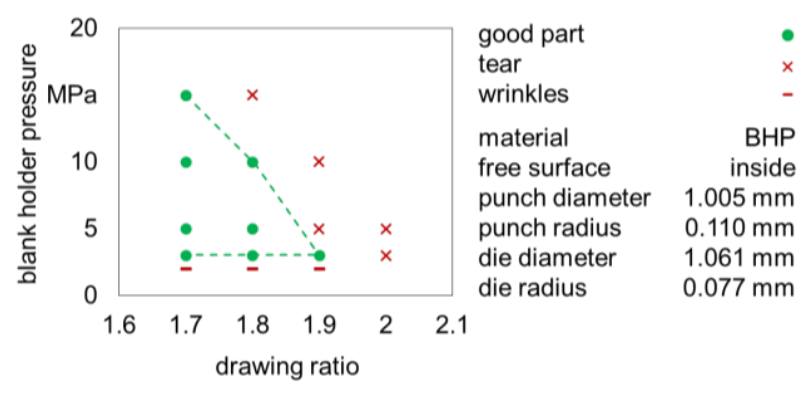

Heinrich 2018

BIAS ID 180275

Fig. 13. Process window of a bimetallic Al-Sc-Zr / stainless steel foil determined in micro deep drawing experiments

A comparison of all deep drawn materials is shown in Figure 14. In case of the bimetallic Al-Sc-Zr / stainless steel foil (BHP) a limiting drawing ratio of 1.9 is achieved independently of the side facing the punch. However, in the monometallic Al-Sc-Zr (MHP) the limiting drawing ratio decreases from 1.8 to 1.7 if the free surface is facing away from the punch.

The maximum punch force achieved during deep drawing experiment is shown in Figure 15. The drawing ratio was set to 1.7 and a blank holder pressure of $5 \mathrm{MPa}$ was used. If the the monometallic Al-Sc-Zr (MHP) is used, no significant difference can be found depending on the side facing the punch. In the case of the bimetallic $\mathrm{Al}-\mathrm{Sc}-\mathrm{Zr}$ / stainless steel foil (BHP) foil, the punch force decreases from $52 \mathrm{~N}$ to $45 \mathrm{~N}$ if the steel side is facing the die. 


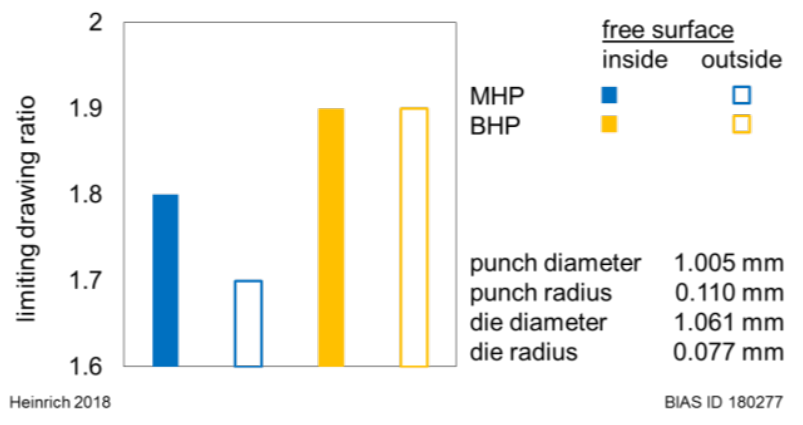

Fig. 14 Limiting drawing ratios determined in micro deep drawing experiments monometallic $\mathrm{Al}-\mathrm{Sc}-\mathrm{Zr}$ foils and a bimetallic Al-Sc-Zr / stainless steel foil produced by HiPIMS

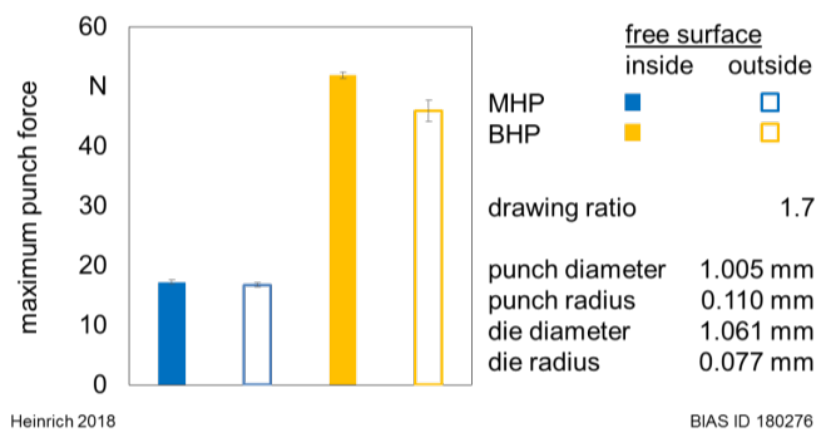

Fig. 15 Maximum punch forces determined in micro deep drawing experiments of monometallic Al-Sc-Zr foils and a bimetallic Al-Sc-Zr/ stainless steel foil

Figure 16 shows a comparison between the inner and outer surface of two cups made of monometallic Al-Sc$\mathrm{Zr}$ (MHP). In the first case (a and c) the free surface was facing the punch. Both inner and outer surface are free of cracks. If the free surface is facing the die (b and d) small cracks can be found on the outer surface. As the maximum punch force does not change in case of the monometallic Al-Sc-Zr (MHP) foil, the difference in limiting drawing ratio depended on the side that is facing towards the die cannot be explained by varying friction. It can however be seen that the porosity has significant influence on the process. If the more porous free surface side is facing towards the die, tears along the initial porosity appear at the area of the punch radius causing the part to fail at a lower limiting drawing ratio. If the bimetallic Al-Sc-Zr / stainless steel foil (BHP) is used, no negative influence on the limiting drawing ratio can be found and the deep drawn cups achieve the same limiting drawing ratio as the substrate material tested in [4].
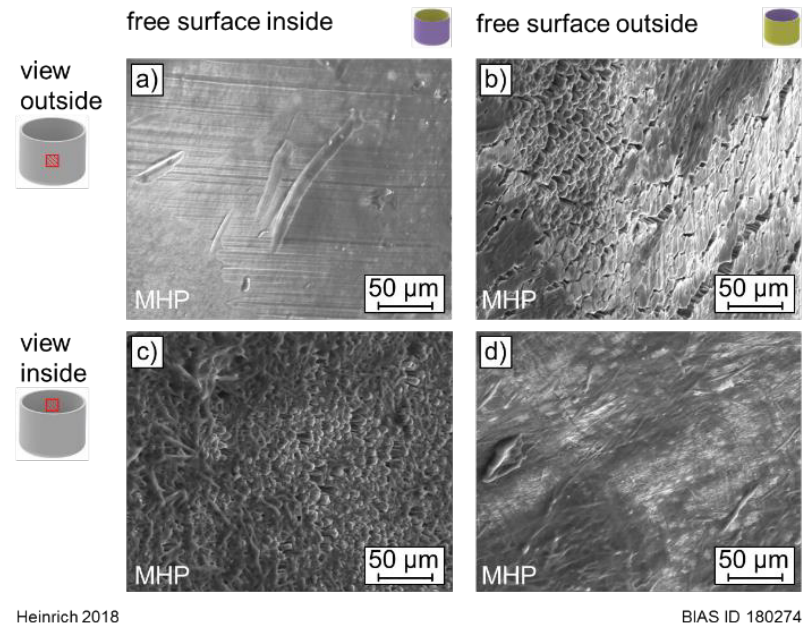

Surface textures of cups made of MHP monometallic foils with a drawing ratio of 1.7 near the bottom edge.

\section{Summary}

In this study, different Al-Sc-Zr monometallic foils and Al-Sc-Zr / stainless steel bimetallic foils were fabricated by using direct current (DC) and high power impulse magnetron sputtering (HiPIMS). Their mechanical properties were investigated by tensile testing. The results of this investigation have shown that the foils sputtered by DCMS show surface porosities, reducing their ductility. Denser foils with better ductility were obtained by increasing the DC target power or by using HiPIMS. Bimetallic Al-Sc-Zr / stainless steel foils show in comparison to monometallic Al-Sc-Zr foils higher yield and tensile strengths and a much higher ductility. By varying the target power and the sputtering mode (DC or HiPIMS) in a same manner as for the monometallic sheet, it was shown that the mechanical properties of the bimetallic sheets vary accordingly to the mechanical properties of the components layers. Variations of the layer thickness of both Al-Sc-Zr and stainless steel have shown that at least the yield and tensile strengths of the bimetallic sheets can be predicted by the rule of mixture as it was already shown by other authors for thick Al / steel bimetallic sheets. The drawability of both a monometallic Al-Sc-Zr foil and a bimetallic Al-Sc-Zr / stainless steel foil produced by HiPIMS were compared in micro deep drawing experiments. Both foils have shown a higher limit drawing ratios than DCMS Al-Zr foils which were previously produced [18]. However, in case of the monometallic foil, different limiting drawing ratios were determined according to the side of the foil (free surface or substrate) facing toward the deep drawing die, which was attributed to surface porosity. If bimetallic Al-Sc-Zr / stainless steel foil is used, the limiting drawing ratio of the substrate material is achieved independently of the orientation of foil.

The authors gratefully acknowledge financial support by DFG (German Research Foundation) for projects A1 B7 and B4 within the Collaborative Research Center SFB 747 Micro Cold Forming - Processes, Characterization and Optimization. 


\section{References}

1. Frank Vollertsen, Micro Metal Forming (Springer, Berlin, Heidelberg, 2013)

2. F. Vollertsen, Z. Hu, H. Schulze Niehoff, C. Theiler, J. Mat. Proc. Tech. 151, 70 (2004)

3. http://www.sfb747.uni-bremen.de

4. G. Behrens, F. O. Trier, H. Tetzel, F. Vollertsen, Int. J. Mater. Form. 9, 253 (2016)

5. G. Brabie, M. Costache, N. Nanu, B. Chirita, Int. J. Mech. Sci. 68, 277 (2013)

6. S. Hadi, A. Kiet Tieu, C. Lu, H. Zhu, Int. J. Mat. Prod. Tech., 47, 175 (2013)

7. J. Royset, N. Ryum, Int. Mat. Rev., 50, 19 (2005)

8. K. Yu, W. Li, S. Li, J. Zhao, Mat. Sci. Eng.A, 368, 88 (2004)

9. V. Neubert, B. Smola, I. Stulikova, A. Bakkar, J. Reuter, Mat. Sci. Eng. A, 464, 358 (2007)

10. A. Cabello Munoz, G. Rückert, B. Huneau, X. Sauvage, S. Marya, J. Mat. Proc. Tech., 197, 337 (2008)

11. S. Iwamura, Y. Miura, Acta Mat., 52, 591 (2004)

12. C. B. Fuller, J. L. Murray, D. N. Seidman, Acta Mater., 53, 5401 (2005)

13. M. Song, Y. He, S. Fang, J. Mater. Eng. Perform., 20, 377 (2011)

14. O. Roder, T. Wirtz, G. Gysler., G. Lutjering, Mat. Sci. Eng. A, 234-236, 181 (1997)

15. http://www.sme.org/MEMagazine/Article.aspx?id=3 $\underline{1841 \& \operatorname{taxid}=1419}$

16. J. Kovac, H.-R. Stock, B. Koehler, H. Bomas, H.-W. Zoch, Surf. Coat. Tech., 215, 369 (2013)

17. R. von Bargen, Z. Hu, H.-W. Zoch, F. Vollertsen, Materialwissenschaft und Werkstofftechnik, 42, 1035 (2011)

18. G. Behrens, J. Kovac, B. Koehler, H. Bomas, H.-W. Zoch, Trans. Nonferrous Met. Soc. China, 22, 268 (2012)

19. D. N. Lee, Y. K. Kim, J. Mat. Sci., 23, 1436 (1988)

20. M. H. Parsa, K. Yamaguchi, N. Takakura, Int. J. Mech. Sci., 43, 268 (2001)

21. H. Takuda, K. Mori, H. Fujimoto, N. Hatta., J. Mat. Proc. Tech., 60, 291 (1996)

22. E. Afshin, M. Kadkhodayan, Mat. Des., 87, 25 (2015)

23. D. Pan, K. Gao, J. Yu, Mat. Sci. Tech., 5, 934 (1989)

24. B. Köhler, H. Bomas, J. Stalkopf, H.-W. Zoch, J. Eng. Integrity Soc., 31, 18 (2011)

25. J. A. Thornton, J. Vac. Sci. Technol. A, 4, 3059 (1986)

26. K. H. Mueller, J. Appl. Phys., 62, 1796 (1987)

27. K. Sarakinos, J. Alami, S. Konstantidinis, Surf. Coat. Tech., 204, 1661 (2010)
28. M. Ohring, Material Science of Thin Films (second edition) (Academic Press, San Diego, San Francisco, New York, Boston, London, Sydney, Tokyo, 2002)

29. S.-H. Choi, K.-H. Kim, K. H. Oh, D. N. Lee, Mat. Sci. Eng A, 222, 158 (1997) 